\title{
Entre lo público y lo privado: acceso y equidad en la educación superior en México y Brasil, 2000-2016
} Between the Public and the Private: Access and Equity in Higher
Education in Mexico and Brazil, 2000-2016

\author{
Alejandro Márquez Jiménez y Armando Alcántara Santuario’
}

\section{Resumen}

Los objetivos del trabajo consisten en analizar en dos países de mediano nivel de desarrollo, el avance que han tenido en términos de la cobertura y la distribución a nivel estatal de las oportunidades de acceso equitativo a la educación superior en el periodo 2000 y 2016. Los países seleccionados, México y Brasil, muestran importantes similitudes en algunas de sus características demográficas, socioeconómicas y educativas. Sin embargo, difieren de manera importante en las estrategias que han seguido en el desarrollo de sus respectivos sistemas de educación superior, en términos de la preponderancia de los sectores público y privado. El análisis se sustenta en pruebas de correlación lineal aplicado a diversos indicadores económicos, sociales y educativos para los años 2000 y 2016. Los resultados muestran que en el periodo considerado ambos países lograron avances en la cobertura, pero tendieron a mantener las inequidades y asimetrías entre las entidades federativas (estados) de ambos países, notándose este efecto en mayor medida en el caso brasileño. En perspectiva, se asume que los resultados del análisis no son concluyentes como para definir cuál de las estrategias seguidas por los países analizados resultan más efectivas para ampliar la cobertura de la educación superior con equidad, dado que en ambos las estrategias utilizadas han resultado ampliamente insuficientes para generar cambios relevantes en la distribución de las oportunidades de acceso a la educación superior a nivel estatal.

\section{Palabras clave}

Políticas públicas, políticas educativas, educación superior, desigualdad y equidad.

\section{Abstract}

The objectives of this article are aimed at analyzing in two countries of medium level of development, the progress they have achieved in student participation and the distribution of opportunities, at the state level, of equitable access to higher education in the period 2000 and 2016. The two countries, Mexico and Brazil, show important similarities in some of their demographic, socioeconomic and educational characteristics. However, they differ significantly in the strategies they have followed in the development of their respective higher education systems, in terms of the preponderance of the public and private sectors. The analysis is based on tests of linear correlation applied to various economic, social and educational indicators for the years 2000 and 2016. The results show that in the period considered both countries achieved advances in student participation, but tended to maintain inequities and asymmetries between the federative entities (states) of both countries, noting this effect to a greater extent in the Brazilian case. In perspective, it is assumed that the results of the analysis are not conclusive to define which of the strategies followed by the countries analyzed is more effective in expanding the level of student participation in higher education with equity, given that in both countries the strategies used have been largely insufficient to generate relevant changes in the distribution of opportunities for access to higher education at the state level.

\section{Keywords}

Public policies, educational policies, higher education, inequality and equity.

\section{Cómo citar/Citation}

Márquez Jiménez, Alejandro; Alcántara Santuario, Armando (2019). Entre lo público y lo privado: acceso y equidad en la educación superior en México y Brasil, 2000-2016. Revista de Sociología de la Educación-RASE, 12 (2), 266-286. http://dx.doi.org/10.7203/RASE.12.2.14873.

1 Alejandro Márquez Jiménez, Universidad Nacional Autónoma de México (UNAM), amj08@unam.mx; Armando Alcántara Santuario, Universidad Nacional Autónoma de México (UNAM), aralsantuario@unam.mx. 


\section{Introducción}

La desigualdad que caracteriza la estratificación social en las actuales sociedades capitalistas se sustenta en la promesa, al menos en principio, de que todos sus miembros tienen la posibilidad de experimentar movilidad social. Aunque ésta puede ser ascendente o descendente, la esperanza de mejorar las condiciones de vida a través de los mecanismos de movilidad social es lo que convence a las amplias mayorías para seguir participando en este sistema social, aceptando las normas que las rigen (Barber, 1978; Giddens, 1995). Al respecto, las teorías funcionalistas y del capital humano, han contribuido a fijar a la educación como uno de los mecanismos más efectivos para superar las barreras estructurales en las sociedades capitalistas, al constituir la puerta de acceso a las posiciones más prestigiosas y mejor remuneradas en el mundo del trabajo. Por ello, en las sociedades contemporáneas las políticas orientadas a expandir y democratizar socialmente el acceso a los diferentes niveles escolares, forma común de referirse a la educación, conllevan un discurso político que sustenta la promesa de que la movilidad social es posible, constituyendo así la base de las creencias sobre un futuro más igualitario (Villa, 2016). Es decir, sustenta la creencia de que mientras se vaya incorporando a aquellos sectores sociales a los niveles educativos a los cuales antes no podían acceder, se logrará la consecuente disminución de la desigualdad social. En razón de ello, un pilar de las modernas políticas gubernamentales es el que tiene como objetivo expandir las oportunidades de acceso a los distintos niveles educativos para la población, siendo la ampliación equitativa de las oportunidades de acceder a la educación superior uno de los logros más importantes de las políticas para el sector educativo.

Este discurso general sobre los beneficios igualadores de la educación se mantiene, a pesar de los cuestionamientos que se han realizado desde distintas vertientes; como, por ejemplo, el relativo a las dificultades que existen para anular las barreras estructurales que impiden el acceso equitativo de los diferentes sectores a la educación superior que, en su momento, sirvieron de sustento a las teorías de la educación como un aparato de reproducción social (Bourdieu y Passeron, [1979] 1996), pero que más recientemente siguen mostrando los trabajos sobre movilidad social intergeneracional (Blanden, 2009; Huerta, 2012); o bien, a las dificultades que actualmente afrontan los jóvenes egresados de la educación superior, principalmente en los países más desarrollados, para acceder a un empleo que les permita mejorar sus condiciones de vida y que ha dado cabida a trabajos relativos al problema de la «sobre educación» (Budría y Moro-Egido, 2009).

Problemas como los anteriores afectan de forma diferente a los países, según los niveles de desarrollo y de cobertura educativa que han alcanzado. Mientras que los menos desarrollados todavía se preocupan por lograr un acceso equitativo a los distintos niveles escolares, principalmente en el nivel superior donde se refleja aún más la desigualdad; en los países desarrollados, que han alcanzado altos niveles de cobertura en la educación superior, el problema se encuentra del lado de los resultados educativos, siendo un problema central el de generar empleos que permitan ocupar de forma adecuada a los egresados de la educación superior.

Dichos problemas, en algún momento fueron enmarcados como parte de los principios que se deberían cubrir desde la perspectiva del enfoque de la igualdad de oportunidades en educación (Latapí, 1993) y acorde con los tiempos, actualmente son considerados como parte de lo que se ha venido conceptualizando como equidad educativa. Al respecto, se dice que las acciones de los Estados Nacionales a través de las políticas educativas deberían asegurar: igualdad en el acceso, igualdad en el trato (referido a la igualdad de recursos y procesos escolares) e igualdad de resultados (en términos de logro académico y desarrollo de capacidades) (Rodríguez, 2008). 
En este sentido, de las tres acepciones de igualdad que acompañan tanto a los discursos de la igualdad de oportunidades como al de la equidad educativa, los países expresan preocupaciones diferentes según su nivel de desarrollo. Así, mientras los menos desarrollados todavía están ocupados en lograr un acceso equitativo, los más desarrollados se preocupan por los resultados de la educación superior.

A partir de lo anterior, los objetivos del presente escrito estriban en analizar en dos países de mediano nivel de desarrollo, el grado de avance que han logrado en términos de la cobertura y la distribución a nivel estatal de las oportunidades de acceso equitativo a la educación superior en el periodo 2000 y 2016. Los dos países seleccionados son México y Brasil, que guardan importantes similitudes en sus características, pero también amplias diferencias en cuanto al desarrollo de sus respectivos sistemas de educación superior. Así, mientras que en México se ha apostado por ampliar las oportunidades de acceso a través de políticas para expandir la oferta de educación superior pública, en Brasil ocurre lo contrario, favoreciendo la expansión de las instituciones de educación superior privadas. Ello ha dado como resultado que en México la matrícula sea mayoritariamente pública (72\%), en tanto que en Brasil sea mayoritariamente privada $(72 \%)$. Por otra parte, en términos de sus similitudes, en años recientes ambos países han puesto en marcha programas de becas para estudiantes de escasos recursos como parte de las estrategias para asegurar el acceso equitativo a la educación superior.

En perspectiva, conocer los avances en la cobertura de la educación superior y en la forma como se han distribuido las oportunidades educativas a nivel estatal, invita a reflexionar y nutrir las discusiones sobre las estrategias seguidas por estos países para ampliar las oportunidades de acceso a la educación superior, tanto en términos del debate entre lo público y lo privado, como en la efectividad de los programas de becas para estudiantes de escasos recursos.

El trabajo se divide en cinco apartados, esta introducción donde se plantea el problema a analizar, un marco contextual orientado a describir algunas de las características que se consideran relevantes en ambos países desde la perspectiva comparativa, un apartado metodológico donde se describen los procedimientos seguidos en la realización del trabajo y las fuentes de información consultadas, le sigue un apartado con los resultados de un análisis a base de correlaciones lineales simples y finalmente, se presentan unas consideraciones finales sobre el trabajo realizado.

\section{Indicadores del contexto demográfico, económico y educativo en México y Brasil}

En este apartado se presenta una serie de indicadores que procuran contextualizar la situación de los dos países a comparar, México y Brasil. Estos indicadores procuran mostrar las similitudes y las diferencias que habría que considerar al interpretar la situación que guardan estos países siguiendo tres vertientes, la demográfica, la económica y la educativa.

Cabe advertir que, aunque con algunas diferencias, uno de los motivos que llevó a seleccionar a estos dos países estriba en que ambos son considerados como países grandes, tanto por el tamaño de su territorio como por su número de habitantes. Si bien, entre los países de América Latina, Argentina ocupa el segundo lugar en cuanto al tamaño de su territorio (2,78 millones de $\left.\mathrm{Km}^{2}\right)$ y México el tercero, el primero tiene una población considerablemente menor a la de los países seleccionados (43,6 millones de habitantes). (Tabla I)

En relación con su dinámica demográfica, aunque Brasil tiene una población considerablemente mayor a la de México, presenta ciertas particularidades en términos demográficos que es bueno considerar, tales 
como una menor tasa de crecimiento, una proporción menor de su población habita en zonas rurales ${ }^{2}$ y tiene un índice de dependencia menor. (Tabla I)

Una tasa menor de crecimiento demográfico brinda ventajas porque mitiga la presión sobre la demanda de bienes y servicios por parte de los nuevos integrantes. Asimismo, tener una menor proporción de la población viviendo en zonas rurales disminuye el compromiso del Estado para atender a la población dispersa en pequeñas localidades, considerando que ello limita generar economías de escala al brindarles bienes y servicios públicos. Aspectos que ocasionan que lo rural se encuentre estrechamente vinculado a carencias y pobreza.

Por su parte, Brasil ha mantenido un índice de dependencia menor ${ }^{3}$ que el de México, lo que implica que su población activa es mayor en comparación con la inactiva, en contraste con México. De este modo, se considera que Brasil puede aprovechar mejor el denominado «bono demográfico» para incentivar su capacidad productiva, sea a través de promover las inversiones en educación y salud, o bien, de incentivar la inversión productiva e incrementar las oportunidades laborales. Esto es algo prioritario que tienen que hacer ambos países, dado que dentro de poco verán crecer paulatinamente el grupo de población de mayor edad (65 años y más). Entre los años 2000 y 2016, este grupo aumentó de $5 \%$ a $8 \%$, en Brasil y de $5 \%$ a $7 \%$, en México.

Tabla I. Indicadores demográficos de México y Brasil, 2000 y 2016

\begin{tabular}{lcrcc}
\hline & \multicolumn{2}{c}{ BRASIL } & \multicolumn{2}{c}{ MÉXICO } \\
\hline & 2000 & 2016 & 2000 & 2016 \\
\hline Superficie $\left(\mathrm{Km}^{2}\right)$ & \multicolumn{2}{c}{8515770} & \multicolumn{1}{c}{1964380} \\
\hline Población (Millones de habitantes) & 173,4 & 207,7 & 97,5 & 127,5 \\
\hline Tasa de natalidad & 2,36 & 1,73 & 2,58 & 2,18 \\
\hline Población rưral como \% de la población total & $19 \%$ & $14 \%$ & $25 \%$ & $20 \%$ \\
\hline Índice de dependencia & 54,3 & 43,6 & 64,7 & 50,9 \\
\hline Porcentaje de la población de 15 a 64 años & $65 \%$ & $70 \%$ & $61 \%$ & $66 \%$ \\
\hline
\end{tabular}

Fuente: Banco Mundial, 2018; INEP, 2018; SEP, 2018; Presidencia de la República, 2017.

En el aspecto económico y social, ambos países guardan importantes similitudes, lo que justifica un tanto su clasificación por el Banco Mundial y la Organización de Cooperación y Desarrollo Económico (OCDE) como sociedades de mediano nivel de desarrollo. Si bien, en cuanto a la magnitud de su Producto Interno Bruto ambos países se ubican entre las mayores economías del Mundo, Brasil ocupando la novena posición y México la quinceava; en lo referente al Producto Interno Bruto por Habitante, estos países pasan a ocupar posiciones que los ubican entre las posiciones 70 y 80 a nivel mundial (Expansión, 2019), caracterizándose como países de mediano nivel de desarrollo económico. (Tabla II)

Entre el año 2000 y 2016, se aprecia que Brasil disminuyó más el nivel de desigualdad en la distribución del ingreso económico que México. El índice de GINI ${ }^{4}$ se redujo 7 puntos porcentuales en Brasil, pasan-

2 La definición de población rural difiere entre países, puede hacer alusión a poblaciones menores a 2000 o 2500 habitantes, o bien, a la población censada fuera de los límites de las áreas urbanas.

3 El índice de dependencia se refiere a la relación entre el grupo de población que se considera en edad activa (15 a 64 años) y los grupos de población que se consideran dependientes porque son muy jóvenes o muy viejos para trabajar ( 0 a 14 años y 65 años y más).

4 El índice de Gini indica la desigualdad en una distribución y adquiere valores entre 0 y 1 o entre 0 y 100 cuando se expresa en porcentaje; cuando los valores se acercan a cero indican que la distribución es más equitativa y mientras más se acercan a 1 o 100, según el caso, indican que la distribución tiende a ser más inequitativa o desigual. 
do de 59 a 51,5; mientras que México solo lo redujo 3 puntos porcentuales, pasando de 51,7 a 48,2. Pero, aunque Brasil disminuyó más la desigualdad, su nivel continúa siendo ligeramente mayor al de México. (Tabla II)

En lo que se refiere al valor del Índice de Desarrollo Humano, que es una medida que integra tres dimensiones de manera conjunta: salud (medida por la esperanza de vida al nacer), educación (con la tasa de alfabetización de adultos y la matriculación bruta en educación primaria, secundaria y terciaria) y vida digna (a través del PIB per cápita). Brasil logró aumentar más el nivel de este último indicador, al pasar de 0,685 a 0,759, entre el año 2000 y 2016; aunque como en el caso de la desigualdad en la distribución del ingreso, su situación sigue siendo menos favorable que en México, en ambos años. (Tabla II).

\section{Tabla II. Indicadores económicos de México y Brasil, 2000 y 2016}

\begin{tabular}{|c|c|c|c|c|}
\hline \multirow[t]{2}{*}{ INDICADORES SOCIOECONÓMICOS Y SOCIALES } & \multicolumn{2}{|c|}{ BRASIL } & \multicolumn{2}{|c|}{ MÉXICO } \\
\hline & 2000 & 2016 & 2000 & 2016 \\
\hline PIB (Miles de millones de dólares PPA) & 1511,1 & 3090,2 & 1037,5 & 2188,6 \\
\hline PIB per cápita, PPA (dólares constantes de 2000) & 8779.3 & 10889,0 & 8997,4 & 9871,7 \\
\hline Índice de GINI de la distribución del ingreso económico & 58,4 & 51,5 & 51,4 & 48,2 \\
\hline Índice de Desarrollo Humano & 0,685 & 0,759 & 0,702 & 0,772 \\
\hline
\end{tabular}

Fuente: Banco Mundial, 2018; INEP, 2018; SEP, 2018; Presidencia de la República, 2017.

En relación con los indicadores de escolaridad, con excepción de los años promedio de escolaridad de la población de 25 y más años, Brasil usualmente presenta mejores indicadores educativos. Así, por ejemplo, tiene tasas brutas de cobertura más alta en la educación media para la población de 15 a 17 años, el tamaño de la población matriculada en educación superior y la tasa bruta de cobertura en educación superior. Aunque en el año 2000, Brasil tenía una cobertura en educación superior más baja que México, para el 2016 ya se encontraba por arriba con una cobertura bruta de 39\% y México de 32\%. Asimismo, en educación superior el gasto por alumno en instituciones de educación superior públicas y privadas ha sido considerablemente más alto en Brasil que en México. (Tabla III).

La diferencia más notable entre ambos países es la configuración de la matrícula en términos de la participación de las instituciones públicas y privadas. Mientras que en Brasil ha predominado la matrícula de instituciones privadas en el periodo considerado (67\% a 78\%), en México la cobertura más alta en este último tipo de instituciones se tuvo en el 2000 (31\%) y posteriormente descendió (28\%) al incentivarse de nueva cuenta el crecimiento de la educación superior pública. Con respecto a este indicador, cabe advertir que estos países históricamente siguieron caminos diferentes en el desarrollo de sus sistemas de educación superior. En México la educación universitaria llegó en la colonia como parte del gobierno español y se mantuvo dentro del ámbito de interés de los grupos gobernantes en las diversas etapas de su historia, relación que se consolida con el triunfo de la Revolución de 1910, entrando a la época moderna y donde ideológicamente el Estado Mexicano asume un discurso favorable a la educación pública (desde la básica hasta la superior) que se mantiene hasta la actualidad (De Ibarrola, 1986; Marsiske, 2006). En contraste, en Brasil la conquista por parte de los portugueses sigue un camino diferente, ellos no se interesan por crear universidades e incluso las prohíben ante el temor de alguna sublevación y prefieren formar sus cuadros profesionales en Portugal, si bien en el país sudamericano se crean algunas escuelas y centros profesionales a cargo de religiosos. Con la independencia de Brasil en 1822, surge el interés por fundar universidades, pero sólo se llegan a crear algunas escuelas, centros y facultades que funcionan de 
forma aislada. Será hasta 1920 cuando se establezca la primera universidad pública en Brasil, a partir de las facultades preexistentes, la Universidad de Rio de Janeiro. Posteriormente, entre 1930 y 1950, existe un renovado interés por crear y consolidar un sistema de universidades públicas, pero estas iniciativas concluirán con el golpe militar de 1964. Los gobiernos militares verán con suspicacia y temor a la universidad pública, ante el temor de brotes subversivos, por lo cual tenderán a favorecer el desarrollo de las instituciones de educación superior privada. Así, hacia la década de los 70, la matrícula de educación superior en Brasil ya sería mayoritariamente privada, hecho que se mantiene hasta la actualidad (García, 2007; Berdugo, 2014; Cruz y Matos, 2017).

En razón de lo anterior, el debate y la predominancia de la educación superior pública o privada en México y Brasil, es un tanto resultado de su historia y muy anterior a las discusiones que se abrirán en las décadas de los 80 y 90, frente a la preeminencia del modelo de desarrollo neoliberal. Un debate que se centrará en la discusión sobre la capacidad y eficiencia del Estado o del Mercado para satisfacer las necesidades de educación superior para la población.

Finalmente, se incluye un indicador que tiene que ver con la orientación que hacia finales de la década de los 90 ha adquirido la acepción de equidad educativa, ajustándose a los cambios que, bajo el marco de las políticas neoliberales, han adquirido las políticas sociales en varios países; un cambio que ha implicado el paso de políticas con una perspectiva universalista (de corte general) en los servicios que brindaba el Estado, hacia la aplicación de políticas de corte focalizado. Asumiendo que bajo este tipo de políticas es posible orientar de manera más precisa los recursos y apoyos del Estado para beneficiar prioritariamente a los grupos identificados como más vulnerables o rezagados (Duhau, 2001).

Bajo este enfoque, en los últimos años se han implementado como parte de las políticas de equidad en la educación superior, programas de apoyo a través de becas estudiantiles para alumnos identificados en situación de vulnerabilidad. En México, este es el caso del Programa Nacional de Becas y Financiamiento para la Educación Superior (PRONABES) que inició en 2001 y que a partir de 2017 recibe el nombre de Programa de Becas de Manutención. Por su parte, en 2004 se implementa en Brasil el Programa Universidad para Todos (ProUni) y el cual se mantiene con el mismo nombre hasta la fecha. En ambos países, las becas se otorgan siguiendo criterios económicos con el fin de que los beneficiarios sean jóvenes de bajos recursos económicos. No obstante, una diferencia que existe entre los dos programas, es que mientras en México las becas solo operan en el caso de las instituciones públicas y los recursos son principalmente para la manutención de los estudiantes, en Brasil el programa de becas incluye a estudiantes de instituciones públicas y privadas, y los recursos de la beca son principalmente para pagar las colegiaturas en estas últimas instituciones. En razón de lo anterior, conocer los alcances que pueden tener estos programas en la ampliación de las oportunidades de acceder a la educación superior en ambos países resulta de interés para ver el alcance de las estrategias seguidas por cada país. (Tabla III).

Tabla III. Indicadores educativos de México y Brasil, 2000 y 2016

\begin{tabular}{|c|c|c|c|c|}
\hline \multirow[t]{2}{*}{ INDICADORES EDUCATIVOS } & \multicolumn{2}{|c|}{ BRASIL } & \multicolumn{2}{|c|}{ MÉXICO } \\
\hline & 2000 & 2016 & 2000 & 2016 \\
\hline Años de escolaridad media de la población de 25 años y más & 5,6 & 7,8 & 6,7 & 8,6 \\
\hline Cobertura de la Educación Media para la población de 15 a 17 años & $75 \%$ & $79 \%$ & $41 \%$ & $76 \%$ \\
\hline Gasto por alumno en IES públicas y privadas (US Dólares PPA)* & 10361 & 11666 & 6074 & 8949 \\
\hline Matrícula en Educación Superior (Miles de alumnos) & 2694,2 & 6554,3 & 1918,9 & 3523,8 \\
\hline
\end{tabular}




\begin{tabular}{|c|c|c|c|c|}
\hline INDICADORES EDUCATIVOS & \multicolumn{2}{|c|}{ BRASIL } & \multicolumn{2}{|c|}{ MÉXICO } \\
\hline Cobertura de la Educación Superior para la población de 18 a 22 años & $16 \%$ & $39 \%$ & $19 \%$ & $32 \%$ \\
\hline Matrícula en IES privadas & $67 \%$ & $72 \%$ & $31 \%$ & $28 \%$ \\
\hline Programa de becas (Fecha de inicio del programa) & \multicolumn{2}{|c|}{2004} & \multicolumn{2}{|c|}{2001} \\
\hline
\end{tabular}

* El gasto por alumno está estimado en dólares según la metodología del poder de paridad adquisitiva (PPA), los datos del año 2000 corresponden a 2002 y los de 2016 a 2014.

**La información sobre los programas de becas en ambos países corresponden a los años de 2006 y 2016

Fuente: Banco Mundial, 2018; OCDE, varios años; INEP, 2018; SEP, 2018; Presidencia de la República, 2017.

En perspectiva, los países a comparar son dos naciones grandes, con historias diferentes en cuanto al desarrollo de sus sistemas de educación superior, con indicadores demográficos un tanto diferentes en cuanto a su dinámica poblacional, pero con un contexto económico y social muy similar que los ubica como países de mediano nivel de desarrollo. En cuanto al tema educativo, sus indicadores son también un tanto similares y en los últimos años han aplicado políticas muy similares para promover la ampliación de la cobertura y la equidad en sus respectivos sistemas de educación superior; si bien la diferencia más marcada entre estos países es la predominancia de las instituciones del sector público (México) o privado (Brasil) como base de sus respectivos sistemas de educación superior.

\section{Metodología}

El análisis que se realiza a continuación tiene por objetivo conocer el tipo de relaciones que se establecen entre una serie de factores ubicados en los ámbitos económico y educativo con respecto a los avances que se presentan en la cobertura de educación superior y en la distribución a nivel estatal de las oportunidades educativas de este nivel en México y Brasil en el periodo comprendido entre los años 2000 y 2016.

Los indicadores incluidos en el análisis se encuentran a nivel estatal, se agrupan en dos dimensiones: económicos y sociales, y educativos, tal como se presenta en la siguiente tabla. Se consideraron los años 2000 y 2016.

\section{Tabla IV. Indicadores incluidos en el análisis}

\begin{tabular}{|c|c|}
\hline INDICADORES ECONÓMICOS Y SOCIALES & CLAVES \\
\hline Producto Interno Bruto per cápita & PIBpc \\
\hline Salario medio de la población ocupada & Sal_M \\
\hline Índice de Desarrollo Humano & IDH \\
\hline MATRÍCULA EN EDUCACIÓN SUPERIOR (MILES DE ALUMNOS) & CLAVES \\
\hline Años promedio de escolaridad de la población de 25 años y más & APESC \\
\hline Cobertura bruta en bachillerato para la población de 15 a 17 años* & CobBach \\
\hline Porcentaje de la matrícula en instituciones de educación superior privadas & MatPriv \\
\hline Porcentaje que representa el número de alumnos beneficiados por los programas de becas para la educación superior con respecto a la matrícula total en educación superior ${ }^{\star \star}$ & B_MTES \\
\hline Cobertura bruta en educación superior para la población de 18 a 22 años* & CobES \\
\hline
\end{tabular}

* En la estimación de la cobertura para el bachillerato o educación media superior y la correspondiente a la educación superior, sólo se consideró a los alumnos del sistema escolarizado.

** En el caso de los programas de becas, los datos corresponden a los años de 2006 y 2016.

Con respecto a los indicadores, conviene aclarar que el dato sobre el Producto Interno Bruto per Cápita se incluye como logaritmo, que es la forma en que se utiliza para el cálculo del Índice de Desarrollo Humano. Por su parte, las tasas brutas de cobertura para la educación media superior fueron estimadas considerando la edad normativa asignada a este tipo de estudios según la Clasificación Internacional 
Normalizada de la Educación (CINE) de la UNESCO (ver Tabla V). En el caso de la educación superior, para hacer comparable la tasa bruta de cobertura en ambos países se decidió tomar al grupo de edad ajustado a la edad normativa (18 a 22 años) marcada por el Programa Sectorial de Educación 2013-2018 del gobierno mexicano (SEP, 2013).

\section{Tabla V. Educación obligatoria y equivalencias de acuerdo a la clasificación internacional normalizada de la educación (CINE, 201 1): México y Brasil}

\begin{tabular}{|c|c|c|c|c|c|c|c|c|c|c|c|c|c|c|c|c|c|c|}
\hline \multicolumn{19}{|c|}{ EDAD } \\
\hline & 0 & 1 & 2 & 3 & 4 & 5 & 6 & 7 & 8 & 9 & 10 & 11 & 12 & 13 & 14 & 15 & 16 & 17 \\
\hline CINE & $\mathrm{NI}$ & $\mathrm{NI}$ & $\mathrm{NI}$ & $\mathrm{NI}$ & $\mathrm{NI}$ & $\mathrm{NI}$ & $P$ & $P$ & $P$ & $P$ & $P$ & $P$ & $M$ & M & M & M & M & $M$ \\
\hline $\begin{array}{l}\text { Educ. } \\
\text { Oblig. }\end{array}$ & & & & 0 & 0 & 0 & 0 & 0 & 0 & 0 & 0 & 0 & 0 & 0 & 0 & 0 & 0 & 0 \\
\hline Nivel & \multicolumn{3}{|c|}{ Inicial } & \multicolumn{3}{|c|}{ Preescolar } & \multicolumn{6}{|c|}{ Primaria } & \multicolumn{3}{|c|}{ Secundaria } & \multicolumn{3}{|c|}{ Media Superior } \\
\hline CINE & $\mathrm{NI}$ & $\mathrm{NI}$ & $\mathrm{NI}$ & $\mathrm{NI}$ & $\mathrm{NI}$ & $\mathrm{NI}$ & $P$ & $P$ & $P$ & $P$ & $P$ & $P$ & M & M & M & M & M & M \\
\hline $\begin{array}{l}\text { Educ. } \\
\text { Oblig. }\end{array}$ & & & & & 0 & 0 & 0 & 0 & 0 & 0 & 0 & 0 & 0 & 0 & 0 & 0 & 0 & 0 \\
\hline Nivel & \multicolumn{4}{|c|}{ Guarderías } & \multicolumn{2}{|c|}{ Preescolar } & \multicolumn{9}{|c|}{ Enseñanza Fundamental } & \multicolumn{3}{|c|}{ Enseñanza Media } \\
\hline
\end{tabular}

Fuente: SITEAL, 2019a; 2019b.

Para el análisis se decidió utilizar únicamente la prueba de correlación líneal de Pearson, puesto que no se busca establecer una relación de causalidad entre los indicadores incluidos en el análisis, dado que se asume que cada uno de ellos es resultado de una relación mucho más compleja donde influyen e interaccionan entre ellos mismos y con respecto a muchos otros factores de los que no es posible conseguir información a nivel estatal. En todo caso, lo que se busca es mostrar la fuerza con que estos indicadores se relacionan entre sí, al igual que el comportamiento de las tasas brutas de cobertura y la distribución de éstas a nivel estatal.

Cabe advertir, que el coeficiente de correlación de Pearson brinda información sobre la fuerza de la relación líneal que se establece entre dos variables cuantitativas y puede adquirir valores entre 1 y - 1 , los coeficientes que se acercan a 1 implican una relación muy fuerte donde ambos indicadores crecen en el mismo sentido y los valores cercanos a -1 indican la existencia de una correlación negativa en la que mientras una variable crece la otra decrece. Los valores cercanos a 0 indican la ausencia de relación entre las variables incluidas en el análisis.

En el mismo análisis se incluyen tres matrices de correlación que toman como eje central los siguientes aspectos: a) el porcentaje que representa el número de alumnos beneficiados por los programas de becas para la educación superior con respecto a la matrícula total en educación superior; b) el porcentaje de la matrícula inscrita en instituciones de educación superior privadas; y c) la tasa bruta de cobertura bruta en educación superior para la población de 18 a 22 años.

Las fuentes de información utilizadas incluyen:

a) Bases de datos e informes internacionales: entre los que se encuentran, la base de indicadores del Banco Mundial (2018), los informes de la Organización para la Cooperación y Desarrollo Económico (OCDE) (varios años), los informes y bases de datos del Programa de las Naciones Unidas para el Desarrollo (PNUD) (2019) e información del sitio Web de Radboud University (2019) sobre los datos a nivel estatal de algunos de los indicadores del Índice de Desarrollo Humano. 
b) Indicadores nacionales para Brasil: se recurrió a las estadísticas generadas por el Instituto Brasileño de Geografía y Estadística (IBGE) (2013; 2019), el Ministerio de Educación de Brasil (MEC) (2019), el Catastro Nacional de Cursos e Institutos de Educación Superior (e-MEC) (2019), el Instituto Nacional de Estudios e Investigaciones Educativas Anísio Teixeira (INEP) (2018) y el sitio Web del PROUNI (2018).

c) Indicadores nacionales para México: se recurrió a información del Instituto Nacional de Geografía y Estadística (INEGI) (2019), del Consejo Nacional de Población (CONAPO) (2017), los informes y estadísticas de la Secretaría de Educación Pública (SEP) (2018; 2019) y anexos estadísticos de los informes de Gobierno (Presidencia de la República, 2017; 2018).

Finalmente, cabe advertir que algunos de los indicadores son resultado de estimaciones propias a partir de la información disponible en los sitios mencionados.

\section{Resultados}

A continuación, se presentan los resultados del análisis de correlación agrupándolos en las tres variables de interés: a) los programas de becas para la educación superior, b) el porcentaje de alumnos que asiste a instituciones de educación superior privadas y c) la tasa bruta de cobertura en la educación superior.

\section{a) Los programas de becas para la educación superior}

Este análisis se realizó con el fin de apreciar la relación que guarda la distribución de las becas a nivel estatal con algunos de los indicadores económicos, sociales y educativos. Considerando que los programas de becas en ambos países constituyen acciones centrales de las políticas para promover la equidad en el acceso a la educación superior, se esperaba que su distribución a nivel estatal mostrara un efecto compensatorio hacia las entidades más rezagadas en los indicadores seleccionados, lo cual implicaría que las acciones de la política de equidad están funcionando adecuadamente a este nivel.

En Brasil, se aprecia que la distribución de las becas vista a través del porcentaje de alumnos becados con respecto a la matrícula total en los estados, no guarda una relación estadísticamente significativa con ninguna de las variables consideradas en el ámbito económico (Producto Interno Bruto per cápita y Salario promedio de la población ocupada) y social (Índice de Desarrollo Humano), o con el educativo (Años promedio de escolaridad y tasa bruta de obertura en la educación media). Lo anterior implica que al menos a nivel estatal, la distribución de las becas del Programa Universidad para Todos (PROUNI) no refleja una orientación compensatoria acorde con los objetivos de generar una mayor equidad entre las entidades más y menos rezagadas en los indicadores considerados. (Tabla VI).

Por su parte, al correlacionar la tendencia de distribución que han seguido los programas de becas en los años de 2006 y 2016 para apreciar si existían cambios, se encontró que Brasil presentaba una correlación positiva $(0,615)$ en los dos años, lo cual implicaba que la distribución en el año 2016 seguía manteniendo el mismo patrón de distribución que en los años iniciales del programa. Sin embargo, al analizar la información con mayor detalle, se percibe que esta correlación era resultado del efecto de un punto distante en el comportamiento de los datos, provocado por el estado de Roraima, que en ambos años fue favorablemente beneficiado por el programa de becas, pues los alumnos beneficiados por el programa de becas en esta entidad representan el 15\% y $19 \%$ del total de alumnos matriculados en educación superior en 2006 y 2016, respectivamente. Siendo esta 
una cifra muy por arriba de los otros estados brasileños. No obstante, al anular el efecto provocado por el estado de Roraima, el coeficiente de correlación desciende a 0,055 y deja de ser significativo estadísticamente. Por lo cual, se concluye que no existe una relación significativa en los patrones de distribución de los programas de becas para la educación superior en Brasil en los dos años considerados. (Tabla VI y gráficas 1 y 3 )

Por el contrario, en México se percibe que la distribución de las becas para la educación superior sí refleja un efecto compensatorio, aunque sólo para el año 2016. En dicho año, la distribución de las becas establece una correlación negativa con el PIB per cápita y con el IDH estatal; lo cual, implica que tendieron a ser más beneficiados por el programa de becas (PRONABES y ahora Becas de Manutención) los estados que presentan una mayor situación de rezago en estos indicadores.

También se estableció una correlación negativa con los años promedio de escolaridad de la población de 25 años y más para los años 2000 y 2016. En cuanto a los patrones de distribución del programa de becas, como en el caso de Brasil, no existe un mismo patrón de distribución en los años considerados. (Tabla VI y gráfica 2).

Tabla VI. Resultados del análisis de correlación entre los programas de becas para la educación superior y los indicares económicos, sociales y educativos, 2000 y 2016

\begin{tabular}{|c|c|c|c|c|}
\hline & \multicolumn{2}{|c|}{ BRASIL } & \multicolumn{2}{|c|}{ MÉXICO } \\
\hline & B_MTES_06 & B_MTES_16 & B_MTES_06 & B_MTES_16 \\
\hline PIBpc_00 & ; & 011 & -348 &,$- 476^{* *}$ \\
\hline PIBpc_16 & 100 & -075 & -293 &,$- 457^{* *}$ \\
\hline Sal_M_00 & ,054 & ,279 &,$- 414^{*}$ & -315 \\
\hline Sal_M_16 & , 110 & ,335 & -270 &,- 169 \\
\hline IDH_00 & ,012 & , 196 &,$- 405^{*}$ &,$- 389 *$ \\
\hline IDH_16 &, 066 & , 194 & -340 & $-424^{*}$ \\
\hline APEsc_00 & ,029 & 244 &,$- 399 *$ &,$- 426^{*}$ \\
\hline APEsc_16 & , 100 & 289 &,$- 366^{*}$ &,$- 353^{*}$ \\
\hline CobBach_00 & ,250 &, 321 & ,015 &,- 009 \\
\hline CobBach_16 & 122 & ,098 &,- 025 &,- 046 \\
\hline B_MTES_06 & 1 &, $615^{\star \star}$ & 1 & ,330 \\
\hline B_MTES_16 &, $615^{\star \star}$ & 1 & ,330 & 1 \\
\hline
\end{tabular}

* Significancia $=0,05$

$* *$ Significancia $=0,01$

Fuentes: estimaciones propias a partir de información de fuentes diversas. 


\section{Gráficas 1, 2 y 3. Correlación entre la distribución de los programas de becas para la educación superior en 2006 y 2016.}

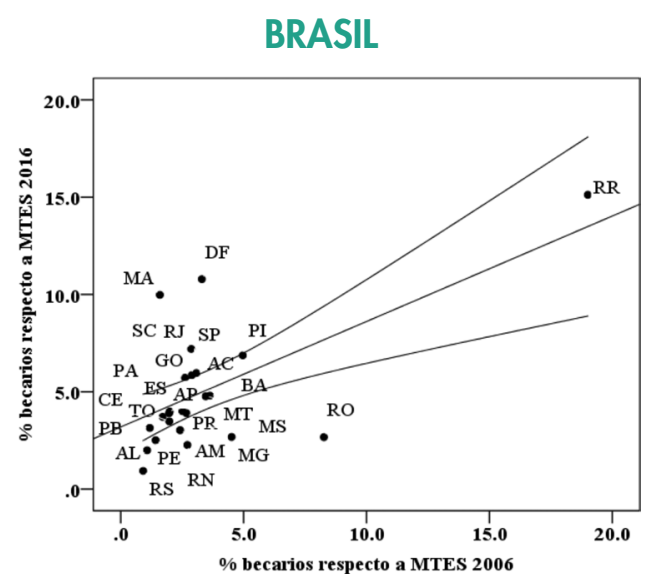

Coeficiente $r=0,615^{* *}$

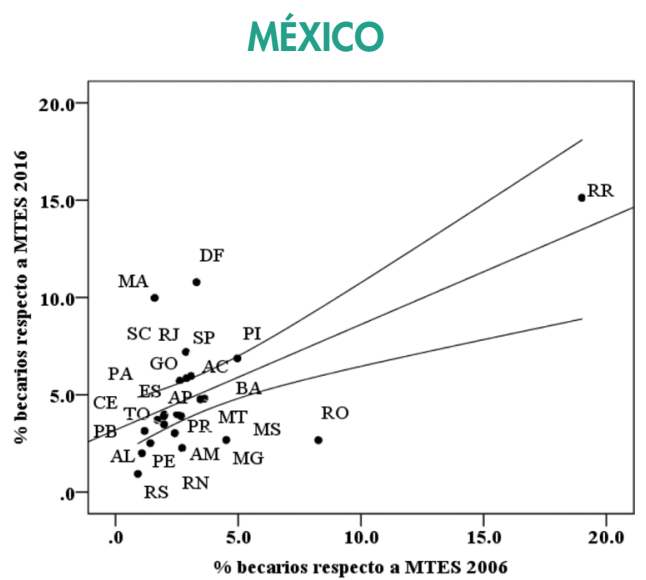

Coeficiente $r=0,330$

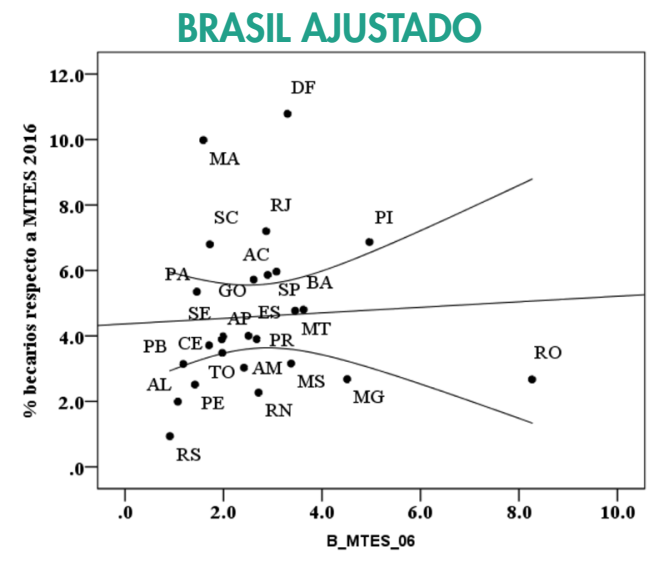

Coeficiente $r=0,055$

$*$ Significancia $=0,05$

$* *$ Significancia $=0,01$

*** En el anexo 1 se encuentra los acrónimos de los estados para Brasil y México.

Fuente: estimaciones propias a partir de diversas fuentes.

En perspectiva, de acuerdo con los resultados obtenidos, es posible señalar que el Programa de Becas para la Educación Superior en México, acorde con su objetivo, refleja un principio compensatorio en la distribución de oportunidades para acceder a la educación superior, dado que tiende a beneficiar en mayor medida a los alumnos de escasos recursos que se ubican en los estados más rezagados en términos económicos, sociales y educativos. Aspecto, que se muestra con mayor claridad en el año 2016. En contraste, en Brasil, los datos analizados no permiten apreciar una tendencia compensatoria en la distribución de las becas para la educación superior.

\section{b)La participación de la matrícula en instituciones de educación superior privadas}

Considerando las diferencias en la forma en que operan los programas de becas en ambos países, este segundo análisis está orientado a ver la relación que guardan las variables de interés en el comportamiento de la matrícula inscrita en instituciones de educación superior privada.

Una relación lógica derivada del principio de mercado que debería estar presente en el desarrollo de la educación superior del sector privado, es que su oferta se establezca de acuerdo a los mayores indicadores económicos y sociales que presentan los estados, tanto en México como en Brasil, dado 
que ello supone la existencia de un mercado potencial para este tipo de educación. Es decir, entre mejor sea la situación económica y social de los estados, es de esperar que la población cuente con mayores recursos y, ello los convierta en demandantes potenciales de la educación privada, la cual usualmente tiene un costo mayor que la pública. En consecuencia, el análisis realizado estaba orientado a corroborar la situación descrita.

Asimismo, era de particular interés conocer el tipo de relación que se establecía entre la distribución de las becas para la educación superior y el crecimiento de la educación superior privada, con el fin de ver si el hecho de que el programa de becas en Brasil apoyara tanto a alumnos del sector público como del privado tenía un cambio en la tendencia de crecimiento de la educación privada; y ver lo mismo para el caso de México, si bien, en este caso el programa solo apoya a los alumnos inscritos en instituciones públicas.

Los resultados encontrados para el caso de Brasil resultan interesantes y muestran la existencia de una correlación positiva entre algunos de los indicadores económicos (PIBpc, Sal M), sociales (IDH) y educativos (APEsc), con respecto al comportamiento de la matrícula inscrita en las instituciones de educación superior privada. Los coeficientes de correlación generalmente son altos (entre 0,538 y 0,841 ) mostrando la relación esperada, es decir, que la cobertura de las instituciones de educación superior privadas tiende a ser mayor conforme aumenta la riqueza de los estados brasileños. (Tabla VII).

Algo que llamó la atención, es que no se encontró (como se esperaba) que existiera relación entre el patrón de distribución de las becas del PROUNI y el comportamiento del porcentaje de la matrícula que es cubierta por las instituciones de educación superior privada; lo cual, probablemente se debe a que el porcentaje de alumnos cubiertos por el programa de becas (entre $3 \%$ y $5 \%$ a nivel nacional en los años 2006 y 2016, respectivamente) es muy pequeño para cambiar la tendencia de comportamiento de la oferta de educación superior privada a nivel nacional. (Tabla 7)

Por su parte, al analizar el grado de correlación que existe en el patrón de distribución del porcentaje de matrícula inscrita en instituciones de educación superior privada en los años 2000 y 2016, se observa que existe una alta correlación positiva $(0,810)$, lo cual, indica que la tendencia en la cobertura cubierta por la oferta de educación privada es muy semejante en ambos años y que probablemente es resultado de variables estructurales vinculadas a la distribución de la riqueza entre los estados brasileños. (Tabla VII y gráfica 4).

Con respecto a la variable de interés, los resultados obtenidos para México fueron muy diferentes a los encontrados en Brasil. En general no se encontró que las variables económicas, sociales y educativas, tuvieran relación con el comportamiento del porcentaje de alumnos matriculados en las instituciones de educación privada a nivel estatal. Solo se estableció una débil correlación positiva y significativa, pero cuyo significado no resulta muy lógico, por lo cual es mejor no prestarle atención. (Tabla VII).

En cuanto a la correlación entre el porcentaje de matrícula inscrita en instituciones de educación superior privada en los años 2000 y 2016, los resultados para México son muy semejantes a los de Brasil, con una alta correlación positiva $(0,829)$ entre ambos años. En este caso, debido a que no se establece relación con otras variables estructurales, se puede decir simplemente que la distribución de la matrícula de las instituciones privadas en los estados de México está influida por los factores 
que han afectado su distribución en el pasado, pero sin poder determinar (a través del análisis realizado) cuáles pueden ser éstos. (Tabla VII y gráfica 5).

Tabla VII. Resultados del análisis de correlación entre el porcentaje de la matrícula inscrita en instituciones de educación superior privada y los indicares económicos, sociales y educativos, 2000 y 2016

\begin{tabular}{|c|c|c|c|c|}
\hline & \multicolumn{2}{|c|}{ BRASIL } & \multicolumn{2}{|c|}{ MÉXICO } \\
\hline & MATPRIV_00 & MATPRIV_16 & MATPRIV_00 & MATPRIV_16 \\
\hline PIBpc_00 &, $841^{* *}$ &, $694^{* *}$ & , 109 & ,236 \\
\hline PIBpc_16 &, $774^{* *}$ &, $555^{* *}$ & $;, 008$ & ,121 \\
\hline Sal_M_00 &, $538^{* *}$ &, $583^{* *}$ & ,272 & ,356* \\
\hline Sal_M_16 &, $608^{* *}$ &, $587^{* *}$ & ,247 &, 316 \\
\hline IDH_00 &, $740^{* *}$ &, $620^{* *}$ &,- 005 & 147 \\
\hline IDH_16 &, $717^{* *}$ &, $584^{* *}$ & -054 & ,082 \\
\hline APEsc_00 &, $702^{* *}$ &, $605^{* *}$ & 057 & 198 \\
\hline APEsc_16 &, $622^{* *}$ &, $557^{* *}$ & $; 040$ & , 123 \\
\hline CobBach_00 & $435^{*}$ & ,367 & -229 & -276 \\
\hline CobBach_16 & -024 & ,086 & ,028 & 067 \\
\hline B_MTES_06 & -343 & ; 163 & - 115 & -281 \\
\hline B_MTES_16 & -257 & -080 & -052 & 010 \\
\hline MatPriv_00 & 1 &, $810^{* *}$ & 1 &, $829^{* *}$ \\
\hline MatPriv_16 &, $810^{* *}$ & 1 & $829^{* *}$ & 1 \\
\hline
\end{tabular}

$*$ Significancia $=0,05$

** Significancia $=0,01$

Fuente: estimaciones propias a partir de diversas fuentes.

Gráficas 4 y 5 . Correlación entre el porcentaje de la matrícula inscrita en instituciones de educación superior privada en 2006 y $2016 * * *$

BRASIL

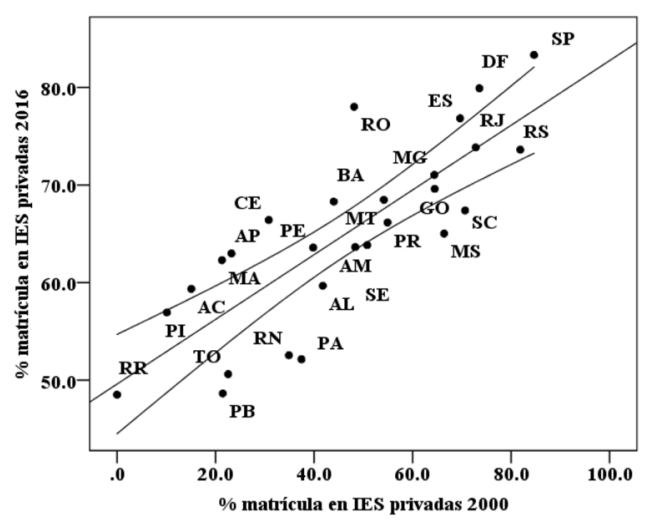

Coeficiente $r=0,810^{* *}$

* Significancia $=0,05$

** Significancia $=0,01$

*** En el anexo 1 se encuentra los acrónimos de los estados para Brasil y México.

Fuente: estimaciones propias a partir de diversas fuentes.
MÉXICO

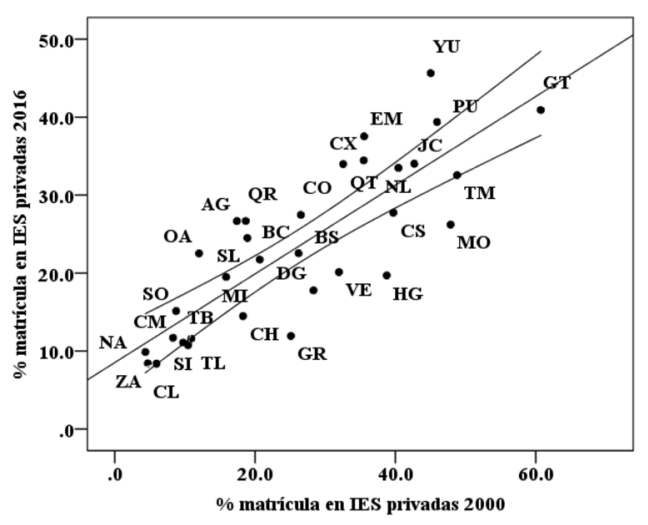

Coeficiente $r=0,829^{* *}$ 


\section{c) La tasa bruta de cobertura en educación superior}

En relación con los patrones de comportamiento de las tasas brutas de cobertura en educación superior, los resultados muestran que éstos están fuertemente correlacionados con el comportamiento de la mayor parte de los indicadores, económicos, sociales y educativos considerados en el análisis. En el caso de variables tales como el PIB per cápita, el salario medio de la población ocupada, el índice de desarrollo humano y los años promedio de escolaridad de la población de 25 años y más, los coeficientes de correlación fluctúan entre 0,666 y 0,895 en Brasil y entre 0,424 y 0,778 en México; lo cual es indicativo de que la relación entre las variables tiende a ser un poco más fuerte para el primer país.

Por lo que se refiere a la relación entre la tasa bruta de cobertura en educación media y la correspondiente a la educación superior, se encontró la tendencia esperada en ambos países, es decir, que las tasas de cobertura en educación media se correlacionan positivamente con las de la educación superior; no obstante, estos resultados resultaron más consistente en México que en Brasil, dado que para el primer país se mantiene en los dos años considerados. En cuanto a Brasil, la relación entre las variables existía en el año 2000, pero no para el 2016; no obstante, se considera necesario tomar este dato con prudencia y procurar corroborarlo con otras fuentes de información diferentes a las consideradas en este análisis. Ello, debido a que en otros trabajos realizados en Brasil (Barreyro, 2008), se ha mostrado la existencia de una fuerte relación entre la cobertura en educación media y la correspondiente a la educación superior.

Por otra parte, solo en el caso de Brasil, el porcentaje de la matrícula inscrita en instituciones de educación superior privada está correlacionado positivamente con la tasa bruta de cobertura en educación superior, con coeficientes de correlación que oscilan entre 0,416 y 0,760. Lo cual indica que según se incrementa el porcentaje de la matrícula inscrita en instituciones de educación superior privada, también aumenta la cobertura de educación superior; ello, es un resultado esperado en razón de la alta participación de la educación superior privada en este país. En México, esta relación no se presenta. (Tabla VIII).

Finalmente, el análisis de correlación realizado con el fin de ver la relación existente en las tasas de cobertura de educación superior en 2000 y 2016, se observa que, en ambos países, los coeficientes de correlación de Pearson son altos y positivos. No obstante, considerando que en ambos casos los estados donde se asienta el gobierno central (DF, en el caso de Brasil y CX, en México) generan el efecto de puntos distantes que pudiesen sesgar el valor de los coeficientes, se decidió correr otro análisis de correlación eliminando a dichas entidades. Como se puede observar en las gráficas 6 a 9, el valor de los coeficientes tiende a disminuir al quitar, en ambos casos, el efecto del gobierno central, si bien, puede considerarse que los coeficientes siguen manteniéndose altos y positivos (coeficiente de 0,687 en el caso de México y de 0,718 en el de Brasil). Lo anterior indica que, en el caso de ambos países, hay pocos cambios en las posiciones que guardan las entidades federativas con respecto a las tasas de cobertura de educación superior. Es decir, aunque todas las entidades ven aumentar sus tasas de cobertura en educación superior durante el periodo considerado, se mantienen las asimetrías que existían entre ellas; esto es, los estados que en 2000 tenían las mayores tasas de cobertura continúan manteniendo su ventaja con el paso del tiempo. (Tabla VIII y gráficas 6 a 9). 
Tabla VIII. Resultados del análisis de correlación entre la tasa bruta de cobertura en educación superior y los indicares económicos, sociales y educativos,

2000 y 2016

\begin{tabular}{|c|c|c|c|c|}
\hline & \multicolumn{2}{|c|}{ BRASIL } & \multicolumn{2}{|c|}{ MÉXICO } \\
\hline & CESUP_00 & CESUP_16 & CESUP_00 & CESUP_16 \\
\hline PIBpc_00 &, $895^{* *}$ &, $710^{* *}$ & $424^{*}$ &, $610^{* *}$ \\
\hline PIBpc_16 &, $832^{* *}$ &, $666^{* *}$ & $456^{* *}$ &, $631^{* *}$ \\
\hline Sal_M_00 &, $723^{* *}$ &, $765^{* *}$ &, $467^{* *}$ &, $633^{* *}$ \\
\hline Sal_M_16 &, $853^{* *}$ &, $867^{* *}$ &, $368^{*}$ &, $513^{* *}$ \\
\hline IDH_OO &, $885^{* *}$ &, $795^{* *}$ &, $678^{* *}$ &, $770^{* *}$ \\
\hline IDH_16 &, $885^{* *}$ &, $823^{* *}$ &, $618^{* *}$ &, $768^{* *}$ \\
\hline APEsc_00 & $857^{* *}$ &, $789^{* *}$ &, $602^{* *}$ &, $737^{* *}$ \\
\hline APEsc_16 &, $814^{* *}$ &, $809 * *$ &, $629^{* *}$ &, $778^{* *}$ \\
\hline CobBach_00 &, $624^{* *}$ &, $660^{* *}$ &, $590^{* *}$ &, $572^{* *}$ \\
\hline CobBach_16 & ,050 & , 183 &, $583^{* *}$ &, $802^{* *}$ \\
\hline B_MTES_06 &,- 047 & ,044 &,$- 405^{*}$ &,$- 359^{*}$ \\
\hline B_MTES_16 & ,089 & 197 & -316 & -298 \\
\hline MatPriv_00 &, $760^{* *}$ &, $431^{*}$ &,- 064 & ,038 \\
\hline MatPriv_16 &, $565^{* *}$ &, $416^{*}$ & -029 & 147 \\
\hline CESup_00 & 1 &, $781^{* *}$ & 1 &, $797^{* *}$ \\
\hline CESup_16 &, $781^{* *}$ & 1 &, $797^{* *}$ & 1 \\
\hline
\end{tabular}

$*$ Significancia $=0,05$

** Significancia $=0,01$

Fuente: estimaciones propias a partir de diversas fuentes.

Gráficas 6, 7, 8 y 9. Correlación entre la tasa bruta de cobertura en educación superior en 2006 y $2016^{* * *}$

BRASIL

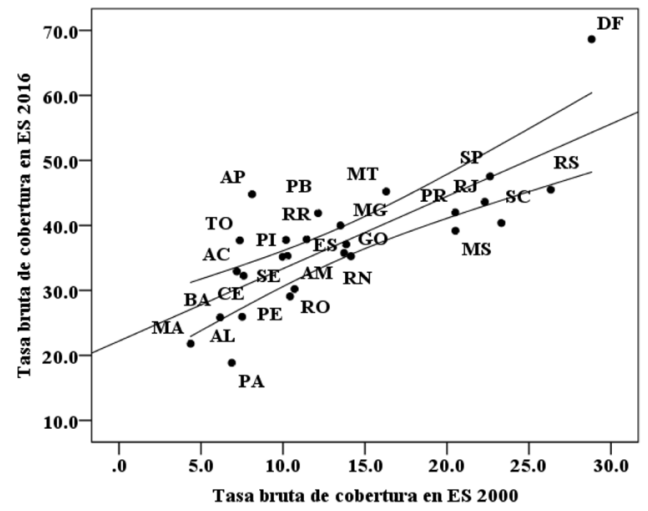

Coeficiente $r=0,781^{* *}$

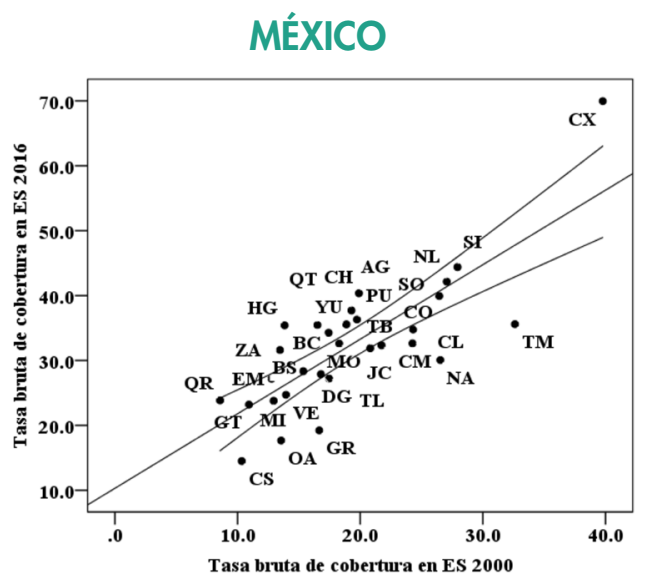

Coeficiente $r=0,797^{* *}$ 


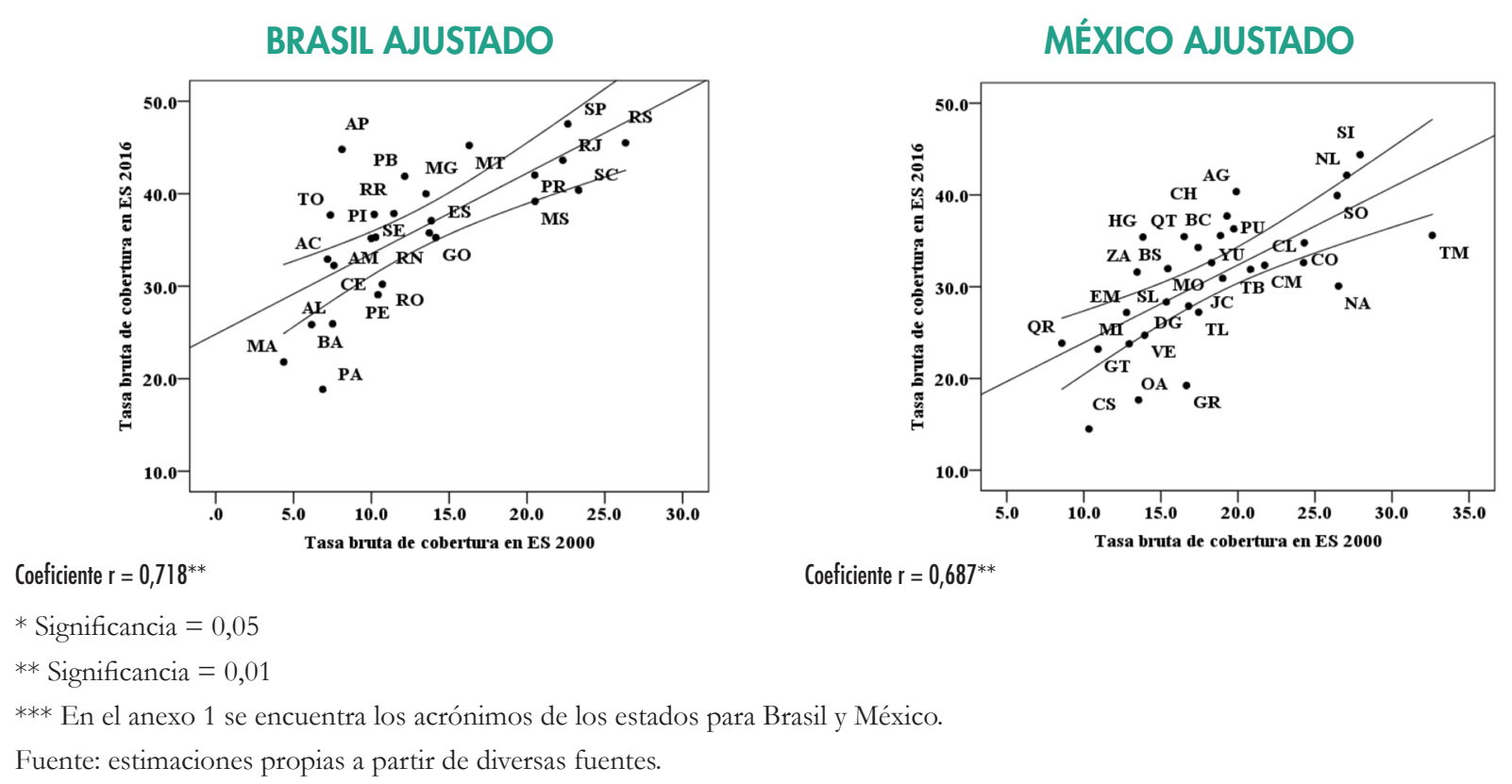

En suma, la tendencia que se observa en los patrones de distribución de los programas de becas en ambos países solo permite considerar que, en el caso de México, existe una tendencia redistributiva que tiende a compensar a los estados que se encuentran en una mayor situación de rezago económico, social y educativo. En Brasil esta tendencia redistributiva a nivel estatal no se aprecia. Por su parte, solo en el caso de Brasil se percibe que existe una consistente relación entre los patrones de distribución del porcentaje de alumnos que asiste a instituciones de educación superior privada y las variables socioeconómicas y educativas consideradas en el análisis, lo cual, probablemente se debe a la mayor participación que históricamente ha tenido este tipo de educación y que la llevó a establecer fuertes vínculos estructurales con diversos factores generadores de desigualdad social. En México, donde la participación de este tipo de educación es menor, esta tendencia no se observa. Finalmente, en ambos países, se observa que la cobertura de la educación superior a nivel estatal mantiene una estrecha relación con la mayoría de las variables y con la tendencia que muestra este mismo indicador en el tiempo, lo cual implica la existencia de factores estructurales que determinan su comportamiento y sobre el que han tenido poca o nula influencia las políticas de equidad educativa instrumentada en ambos países para cambiar esta situación.

\section{Consideraciones finales}

La igualdad de oportunidades para acceder a la educación y principalmente a la de nivel superior, forma parte importante del discurso ideológico que acompaña a los estados capitalistas modernos, pues juega un papel fundamental en la estabilidad social, al constituir a la educación como uno de los mecanismos que posibilitan la movilidad social. Mecanismo al que deberían poder acceder todos los miembros de la sociedad, para que, con base a su esfuerzo y capacidades, puedan aspirar a una vida mejor.

En este sentido, las políticas y estrategias instrumentadas por los países para ampliar equitativamente las oportunidades de acceso a la educación superior, brinda legitimidad al sistema social imperante y a los gobiernos que las aplican. En razón de ello, en este trabajo se consideró importante analizar las estrategias seguidas por dos países de mediano nivel de desarrollo para distribuir equitativamente las oportunidades de acceso a la educación superior. 
Se tomó como marco de comparación la distribución estatal de las oportunidades de acceso a la educación superior y la relación que guarda con dos estrategias seguidas por los países seleccionados. Una, vista a través de la participación de los sectores público y privado en la atención de la matrícula de educación superior; y la otra, a través de los programas de becas que, en años recientes, fueron puestos en marcha en ambos países como parte de las políticas orientadas a promover el acceso equitativo a la educación superior.

En términos generales, se puede señalar que los resultados obtenidos del análisis no son del todo concluyentes, aunque sí suficientes para reflexionar acerca del debate existente entre lo público y lo privado como base del desarrollo de los sistemas de educación superior, así como sobre los alcances y limitaciones que presentan las políticas de apoyo focalizado (a través de los programas de becas) para promover el acceso equitativo a la educación superior.

En cuanto al primer aspecto, Brasil, donde la mayor parte de la matrícula es atendida por instituciones privadas, presentó un mayor avance en cuanto al número de alumnos matriculados y en relación con la cobertura de la educación superior en el periodo considerado (2000 y 2016). Estos resultados invitan a pensar que la estrategia orientada a favorecer la expansión de la educación superior privada es mejor. No obstante, cabe resaltar que la diferencia entre Brasil y México (donde la mayor parte de la matrícula es pública) no es muy grande; pero además, según las evidencias derivadas del trabajo, el avance logrado por Brasil en los indicadores señalados ha tendido (en mayor medida que México) a mantener las desigualdades y asimetrías en el acceso a la educación superior preexistentes entre los estados brasileños.

Otro aspecto a considerar con respecto al debate entre lo público y lo privado como base de la expansión de la educación superior, son los recurrentes cuestionamientos que han realizado algunos investigadores brasileños (ver por ejemplo: Stallivieri, 2007; Barreyro y Oliveira, 2015) sobre la laxitud que ha tenido el gobierno brasileño para supervisar la «calidad» de las instituciones de educación superior privada, que es sobre las que descansa la mayor parte de la matrícula de educación superior. Lo anterior, hace suponer que la expansión de la educación superior en Brasil se ha realizado a costa de sacrificar la calidad educativa. No obstante, estas opiniones sólo sirven para mantener abierto el marco de reflexión dado que sería necesario contar con indicadores que reflejen la «calidad» de la educación que brindan las diversas instituciones de educación superior al interior de cada país y entre países, para tomar una posición concluyente al respecto. Hasta la fecha, sin embargo, este tipo de información no existe.

En cuanto a la estrategia para promover la equidad en el acceso a la educación superior a través de la implementación de programas de becas, los resultados obtenidos muestran que en ambos países los programas han resultado insuficientes para subsanar las inequidades existentes en la distribución de oportunidades educativas, dado que no se perciben cambios sustanciales en relación con las tendencias inequitativas que caracterizan el acceso a la educación superior en ambas naciones. Si bien, cabe resaltar que, en México, aunque insuficientes para cambiar las tendencias generales en los patrones de acceso a la educación superior, en la distribución de las becas se logra apreciar un principio compensatorio que tiende a beneficiar en mayor medida a las entidades menos desarrolladas; relación que no se percibe en la distribución de las becas en el caso brasileño.

Finalmente, cabe destacar que aun cuando en las dos sociedades se notan avances en los indicadores considerados en el análisis, para el periodo examinado, dichos avances han tendido a mantener las inequidades y asimetrías estructurales que preexistían en los estados de ambos países; si bien, este efecto es más 
notorio en Brasil. En este sentido, se puede decir que la cobertura de la educación superior se encuentra estrechamente vinculada a los factores estructurales que generan la desigualdad social en cada país y, en razón de ello, para lograr cambios en la distribución de las oportunidades de acceso a la educación superior, se requiere implementar medidas más contundentes para promover la equidad en el acceso a la educación superior.

\section{Referencias bibliográficas}

Banco Mundial. Indicadores del desarrollo mundial (en línea). http://databank.bancomundial.org/data/ reports.aspx?source $=$ Indicadores $\% 20 \mathrm{del} \% 20$ desarrollo $\% 20$ mundial, consultado el 1 de marzo de 2018.

Barber, Bernard (1978). Estratificación social. México: FCE.

Barreyro, Gladys Beatriz (2008): "La educación superior en Brasil: raza, renta y escuela media como factores de desigualdad”. Revista de la Educación Superior, 37 (146), 53-64.

Barreyro, Gladys Beatriz y Oliveira Costa, Fábio Luciano (2015): "Las políticas de educación superior en Brasil en la primera década del siglo xxI. Algunas evidencias sobre impactos positivos en la equidad”. Revista Mexicana de Investigación Educativa, 20 (64), 17-46.

Berdugo Gómez de la Torre, Ignacio (2014): “La Educación Superior en Brasil. 1. Desde la Independencia al Golpe Militar de 1964”. Revista de Estudios Brasileños, 1 (1), 113-130.

Blanden, Jo (2009). How Much Can We Learn From International Comparisons Of Intergenerational Mobility? London: Centre for the Economics of Education, London School of Economics (en linea). http://cee.lse.ac.uk/ceedps/ceedp111.pdf, consultado el 25 de junio de 2018.

Bourdieu, Pierre y Passeron, Jean-Claude [1979] (1996). La reproducción. Elementos para una teoría de la enseñanza. México: Distribuciones Fontamara.

Budría, Santiago y Moro-Egido, Ana I. (2009): “The overeducation phenomenon in Europe". Revista Internacional de Sociología (RIS), 67 (2), 329-345.

Consejo Nacional de Población (CONAPO) (2017). Estimaciones y Proyecciones de Población por Entidad Federativa, 1990-2010 y 2010-2030 (en línea). http://www.conapo.gob.mx/es/CONAPO/Proyecciones_Datos, consultado el 1 de marzo de 2018.

Cruz Pinto, Ana Cristina y Matos, Maria Almerinda de Souza (2017): “La educación superior en Brasil: un recorrido histórico". Revista Multidisciplinar Científica Centro del Conocimiento, 1 (6), 387-402.

De Ibarrola, María (1986). La educación superior en México. Caracas: CRESALC-UNESCO.

Duhau, Emilio (2001). "Política social, pobreza y focalización. Reflexiones en torno al programa de educación, salud y alimentación” en Alicia Ziccardi (Comp.). Pobreza, desigualdad social y ciudadanía. Los limites de las politicas sociales en América Latina. Buenos Aires: CLACSO.

e-MEC (2019). Cadastro Nacional de Cursos e Instituições de Educação Superior (en línea). http:/ / emec. mec.gov.br/, consultado el 20 de febrero de 2019.

Expansión (2019). Datos Macro, FMI-Fondo Monetario Internacional (en línea). https://datosmacro. expansion.com/paises/grupos/fmi, consultado el 13 de marzo de 2019. 
García Guadilla, Carmen (2007): "Financiamiento de la educación superior en América Latina". Sociologias, (17), 50-101 http://www.scielo.br/pdf/soc/n17/a04n17.pdf, consultado el 20 de febrero de 2018.

Giddens, Anthony (1995). Estratificación y estructura de clase. Sociología, Madrid: Alianza, Textos Universitarios.

Huerta Wong, Juan Enrique (2012): “El rol de la educación en la movilidad social de México y Chile. ¿La desigualdad por otras vías?”. Revista Mexicana de Investigación Educativa, 17 (52), 65-88.

Instituto Brasileiro de Geografia e Estatistica (IBGE) (2019). Estatisticas (en línea). https://www.ibge. gov.br/, consultado el 04 de marzo de 2019.

Instituto Brasileiro de Geografia e Estatistica (IBGE) (2013). Nas vidas do Brasil a vida de um país. Projeção da população 2013 (en línea). https://ww2.ibge.gov.br/home/estatistica/populacao/projecao_da_populacao/2013/default_tab.shtm, consultado el 1 de marzo de 2018.

Instituto de Información Estadística y Geográfica (INEGI). Banco de información económica (en línea). https://www.inegi.org.mx/sistemas/bie/, consultado el 7 de abril de 2019.

Instituto Nacional de Estudos e Pesquisas Educacionais Anísio Teixeir (INEP). Sinopses Estatísticas da Educação Superior - Graduação (en línea). http://inep.gov.br/sinopses-estatisticas-da-educacaosuperior, consultado el 3 de abril de 2018.

Latapí, Pablo (1993): "Reflexiones sobre la justicia en la educación”. Revista Latinoamericana de Estudios Educativos, XXIII (2), 9-41.

Marsiske, Renate (2006): “La universidad de México: Historia y Desarrollo”. Revista Historia de la Educación Latinoamericana, 8, 11-34.

Ministério da Educação (2019). Varias fuentes (en línea). http://portal.mec.gov.br/index.php, consultado el 19 de febrero de 2019.

OECD. Education at a Glance, de 2005 a 2017 (en linea). http://www.oecd.org/education/education-ata-glance-19991487.htm, consultado el 20 de marzo de 2018.

Presidencia de la República (2017). $5^{\text {to }}$ Informe de Gobierno 2016-2017, Anexo Estadístico, 2017 (en línea). http://framework-gb.cdn.gob.mx/quintoinforme/5IG_ANEXO_FINAL_TGM_250818.pdf, consultado el 14 de febrero de 2018.

Presidencia de la República (2018). 6 ${ }^{\text {to }}$ Informe de Gobierno 2017-2018, Anexo Estadístico, 2018 (en línea). https://datos.gob.mx/busca/dataset/sexto-informe-de-gobierno, consultado el 20 de marzo de 2019.

PNUD (varios años). Informes sobre el desarrollo humano 2000 a 2018 (en línea). https://www.undp. org/, consultado el 20 de febrero de 2019.

PROUNI. Dados e Estatísticas, Quadros Informativos, Bolsas ofertadas por Unidade da Federação, 2018 (en línea). http://prouniportal.mec.gov.br/dados-e-estatisticas/9-quadros-informativos, consultado el 2 de abril de 2018.

PROUNI. O Programa, 2018. Disponible en http://prouniportal.mec.gov.br/o-programa, consultado el 10 de marzo de 2018. 
Radboud University (2019). Global_Data_Lab. Subnational Human Development Index (SD-2019) (en línea). https://globaldatalab.org/shdi/, consultado el 18 de abril de 2019.

Rodríguez, Carlos Rafael (2008): "Equidad en la educación en México. Propuesta de un sistema de indicadores". Revista Perspectivas Sociales, 10 (2), 55-79.

Secretaría de Educación Pública (SEP) (2013). Programa Sectorial de Educación 2013-2018. México: Secretaría de Educación Pública.

Secretaria de Educación Pública (SEP). (2018). Sistema Nacional de Información Estadística Educativa, Indicadores y Pronósticos Educativos (en línea). http://www.snie.sep.gob.mx/indicadores.html, consultado el 1 de marzo de 2018.

Secretaria de Educación Pública (SEP). (2019). Sistema Nacional de Información Estadística Educativa, Estadísticas Educativas (en línea). http://www.planeacion.sep.gob.mx/, consultado el 10 de marzo de 2019.

SITEAL (2019a). Perfiles del país. República Federativa del Brasil (en línea). http://www.publicaciones. siteal.iipe.unesco.org/perfiles-de-pais/18/republica-federativa-del-brasil, consultado el 20 de marzo de 2019.

SITEAL (2019b). Perfiles del país. Estados Unidos Mexicanos (en línea). http://www.publicaciones.siteal. iipe.unesco.org/perfiles-de-pais/19/estados-unidos-mexicanos, consultado el 20 de marzo de 2019.

Stallivieri, Luciane (2007). El sistema de educación superior de Brasil: características, tendencias y perspectivas, Universidades, (34), 47-61 (en línea). https://www.ses.unam.mx/curso2017/bibliografia/Stallivieri $\% 202007 . \% 20 \mathrm{El} \% 20$ sistema $\% 20 \mathrm{de} \% 20$ educaci $\% \mathrm{C} 3 \% \mathrm{~B} 3 \mathrm{n} \% 20$ superior $\% 20 \mathrm{de} \% 20 \mathrm{Brasil} . \% 20$ Caracter\%C3\%ADsticas, $\% 20$ tendencias $\% 20 y \% 20$ perspectivas.pdf, consultado el 25 de abril de 2018.

Villa Lever, Lorenza (2016): "Educación superior, movilidad social y desigualdades interdependientes". Universidades, (68), 51-64.

\section{Notas biográficas}

Alejandro Márquez Jiménez es Doctor en Educación por la Universidad Autónoma de Aguascalientes, México. Investigador del Instituto de Investigaciones sobre la Universidad y la Educación de la UNAM.

Armando Alcántara Santuario es Doctor en Educación por la Universidad de California, Los Ángeles. Investigador del Instituto de Investigaciones sobre la Universidad y la Educación de la UNAM. 


\section{Anexos 1}

Acrónimos de las entidades federativas en México y Brasil

\begin{tabular}{|c|c|c|c|c|}
\hline & \multicolumn{2}{|c|}{ MÉXICO } & \multicolumn{2}{|c|}{ BRASIL } \\
\hline & ACRÓNIMOS & ENTIDADES FEDERATIVAS & ACRÓNIMOS & ENTIDADES FEDERATIVAS \\
\hline 1 & $A G$ & Aguascalientes & $A C$ & Acre \\
\hline 2 & $B C$ & Baja California & $\mathrm{AL}$ & Alagoas \\
\hline 3 & BS & Baja California Sur & AM & Amazonas \\
\hline 4 & $C M$ & Campeche & AP & Amapá \\
\hline 5 & $\mathrm{CO}$ & Chiapas & BA & Bahía \\
\hline 6 & $\mathrm{Cl}$ & Chihuahua & CE & Ceará \\
\hline 7 & CS & Coahuila & DF & Distrito Federal \\
\hline 8 & $\mathrm{CH}$ & Colima & ES & Espírito Santo \\
\hline 9 & $C X$ & Ciudad de México & GO & Goiás \\
\hline 10 & $D G$ & Durango & MA & Maranhão \\
\hline 11 & GT & Guanajuato & MG & Minas Gerais \\
\hline 12 & GR & Guerrero & MS & Mato Grosso do Sul \\
\hline 13 & $H G$ & Hidalgo & MT & Mato Grosso \\
\hline 14 & $J C$ & Jalisco & PA & Pará \\
\hline 15 & $E M$ & Estado de México & $\mathrm{PB}$ & Paraíba \\
\hline 16 & MI & Michoacán & PR & Paraná \\
\hline 17 & MO & Morelos & PE & Pernambuco \\
\hline 18 & NA & Nayarit & $\mathrm{Pl}$ & Piauí \\
\hline 19 & $\mathrm{NL}$ & Nuevo León & RJ & Río de Janeiro \\
\hline 20 & $O A$ & Oaxaca & RN & Rio Grande do Norte \\
\hline 21 & PU & Puebla & RO & Rondônia \\
\hline 22 & QT & Querétaro & RR & Roraima \\
\hline 23 & QT & Quintana Roo & RS & Rio Grande do Sul \\
\hline 24 & $S L$ & San Luis Potosí & SC & Santa Catarina \\
\hline 25 & $\mathrm{SI}$ & Sinaloa & SE & Sergipe \\
\hline 26 & SO & Sonora & $S P$ & São Paulo \\
\hline 27 & TB & Tabasco & TO & Tocantins \\
\hline 28 & $T M$ & Tamaulipas & & \\
\hline 29 & $\mathrm{TL}$ & Tlaxcala & & \\
\hline 30 & VE & Veracruz & & \\
\hline 31 & YU & Yucatán & & \\
\hline 32 & $Z A$ & Zacatecas & & \\
\hline
\end{tabular}

